\title{
Wireless Sensor for Temperature and Flux Measurements in an Axial Flux Machine
}

\author{
Andreas Bock ${ }^{1}$, Dian Liu ${ }^{2}$, Jürgen Funck ${ }^{1}$, Artur Giedymin ${ }^{3}$, Richard Burke ${ }^{2}$, Clemens Gühmann ${ }^{1}$ \\ ${ }^{1}$ TU Berlin, Chair of Electronic Measurement and Diagnostic Technology, Sekr. EN13, \\ Einsteinufer 17, 10597 Berlin, Germany, \\ juergen.funck@tu-berlin.de \\ ${ }^{2}$ Department of Mechanical Engineering, University of Bath, Bath BA2 7AY, UK \\ ${ }^{3}$ TU Berlin, Electric Drives Group, Sekr. EM4, Einsteinufer 11, 10587 Berlin, Germany
}

\begin{abstract}
In recent years there has been an increasing interest into electric and hybrid electric vehicles. Axial flux permanent magnet (AFPM) machines appear to be very promising for these applications. To fully exploit the potential of the electric machine to provide short burst of mechanical power higher than the rated continuous power, highly accurate dynamic models of its thermal behavior are needed. To parameterize and validate these models the temperatures and magnetic flux densities on the rotor need to be measured during operation. This contribution presents the design and implementation of a smart wireless sensor for this purpose. An inductive power supply enables a dependable, continuous operation of the sensor without the need for batteries. Signal processing on the wireless sensor ensures an efficient use of the available wireless bandwidth. The mechanical integration of the sensor into the motor as well as its connection to an existing motor test bench are discussed. The operation of the sensor is verified during experiments in a climate chamber as well as on a motor test bench. The presented wireless sensor enables unprecedented measurements at an AFPM machine and can be easily extended with additional sensor in future experiments.
\end{abstract}

Key words: wireless sensor, smart sensor, inductive power transmission, axial flux permanent magnet machine, electric vehicle

\section{Introduction}

In recent years, rises in oil prices and ever stricter vehicle emission standards have led to an increasing interest into electric and hybrid electric vehicles. Axial flux permanent magnet (AFPM) machines appear to be very promising for these applications as they have a high power density and can be built very compact in size [1]. Their thermal management is crucial for extracting the maximum mechanical power from a motor without compromising its lifetime. Electric drives will have a rated maximum power which can be achieved for short bursts but also a significantly lower continuous power below which overheating is not a problem. As the difference between this continuous and maximum power can be significant, it is important to understand the thermal behavior of the complete motor in order to exploit its transient ability when running in a hybrid powertrain. This understanding can be integrated into the powertrain controller development through a thermal model, however to parameterize and validate such a model temperatures on both stator and rotor as well as the flux density need to be measured during operation.

This contribution presents the results of a joined project between the Chair of Electronic Measurement and Diagnostic Technology at the TU Berlin and the Department of Mechanical Engineering at the University of Bath. It describes the design and implementation of a smart wireless sensor that measures the temperatures and the magnetic flux density on the rotor. Section 2 describes the significance of the temperature and magnetic flux for the operation of an AFPM machine. The target system that the wireless sensor is designed for is introduced in section 3. The design of the wireless sensor is given in section 4. Finally, experiments to validate the sensor are presented in section 5 .

\section{Thermal Behavior of Axial Flux Ma- chines}

The permanent magnet (PM) is the most vital component in a PM machine. It defines the motor performance since the magnetic flux linkage inside the air gap determines conversion between electric energy and mechanical 
work. However, rare earth magnets are very delicate when their temperature is beyond their thermal limit. The magnetic properties can significantly vary or may even permanently be removed, i.e. demagnetized, when it is over heated. The two key parameters of magnets, the remnant flux density $(\mathrm{Br})$ and the coercive field strength $(\mathrm{Hc})$, decrease when temperature is climbing which indicates the magnet is losing strength in the free air and becomes easier to be demagnetized when exposed in an opposite magnetic field.

When the motor is air cooled and both stator and rotor are enclosed in the casing as the motor being used in this project, the operating environment is even worse from a thermal perspective. So, it is very important to monitor and analyze the magnets flux density change regarding to its thermal condition. Having the ability to measure the magnet's temperature and air gap flux on-the-fly also gives the opportunity to further increase the accuracy of the electromagnetic model to predict the performance and that of the thermal model to improve the cooling of the PM machine.

\section{Target System}

\subsection{Axial Flux Machine}

The motor being used in this project is a threephase PM synchronous machine. The specifications are given in Tab. 1.

Tab. 1: Parameters of axial flux machine

\begin{tabular}{lr}
\hline \multicolumn{1}{c}{ Parameter } & Value \\
\hline Pole pairs & 4 \\
Rated power $(\mathrm{W})$ & 1500 \\
Rated speed (rpm) & 4500 \\
Rated torque (Nm) & 3.18 \\
Rated efficiency (\%) & 89 \\
Motor casing diameter (mm) & 200 \\
Casing axial length (mm) & 55 \\
\hline
\end{tabular}

Fig. 1 shows a cross section of the original machine. There is already a $3.6 \mathrm{~mm}$ gap between the backside of the rotor and the motor casing that can be enlarged with only minimal modifications. Therefore this is the most suitable place to put additional sensor circuitry.

\subsection{Test Bench}

On the test bench the AFPM machine is powered from an inverter with a DC input voltage of $48 \mathrm{~V}$. An induction motor is used as the dyna- mometer working against the axial flux machine. All three phase' current and terminal voltage are logged by a high speed power analyzer. The whole test bed is controlled by a system from Sierra CP Engineering. It also has the ability log real time physical measurements like the test machine shaft speed, torque, stator coil temperature, etc. via a CAN-bus interface.

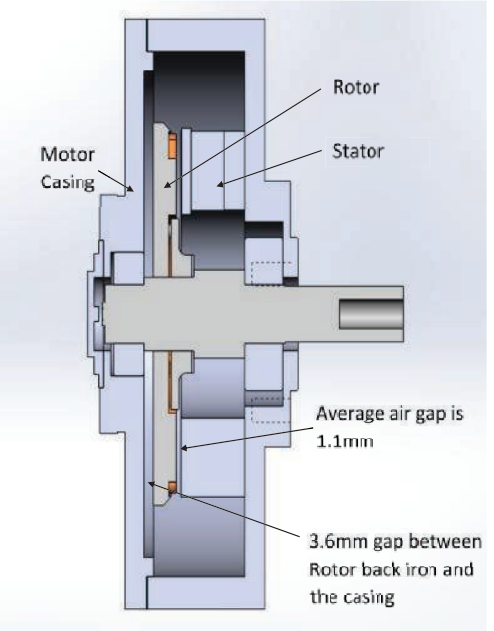

Fig. 1. Cross-section of the original axial flux machine

\section{Design of Sensor}

The goal was to build a sensor that measures the temperature of the rotor at three different locations as well as the magnetic flux density while the motor is in operation. The sensor should be able to withstand temperatures of up to $100{ }^{\circ} \mathrm{C}$ as well as the centrifugal forces inside the motor. In order to affect the motor as little as possible the sensor should be lightweight and have a well balanced mass distribution. To ease the integration of the sensor into the test bench described in section 3.2 the sensor data should be sent continuously to its CAN-bus during the experiments.

Based on the good experiences made with a wireless temperature sensor for an automotive clutch described in [2], it was decided to digitize the data directly on the sensor and to transmit it wirelessly. This avoids the use of error-prone slip-rings and prevents data distortion during transmission. Battery based power supplies for wireless sensors, like the one in [2], have the disadvantage that they can only provide a limited amount of energy and are also quite sensitive to high temperatures and mechanical vibrations. Therefore it was decided to use an inductive power supply for the sensor similar to that described in [3]. Fig. 2 shows the structure of the entire wireless sensor system. 


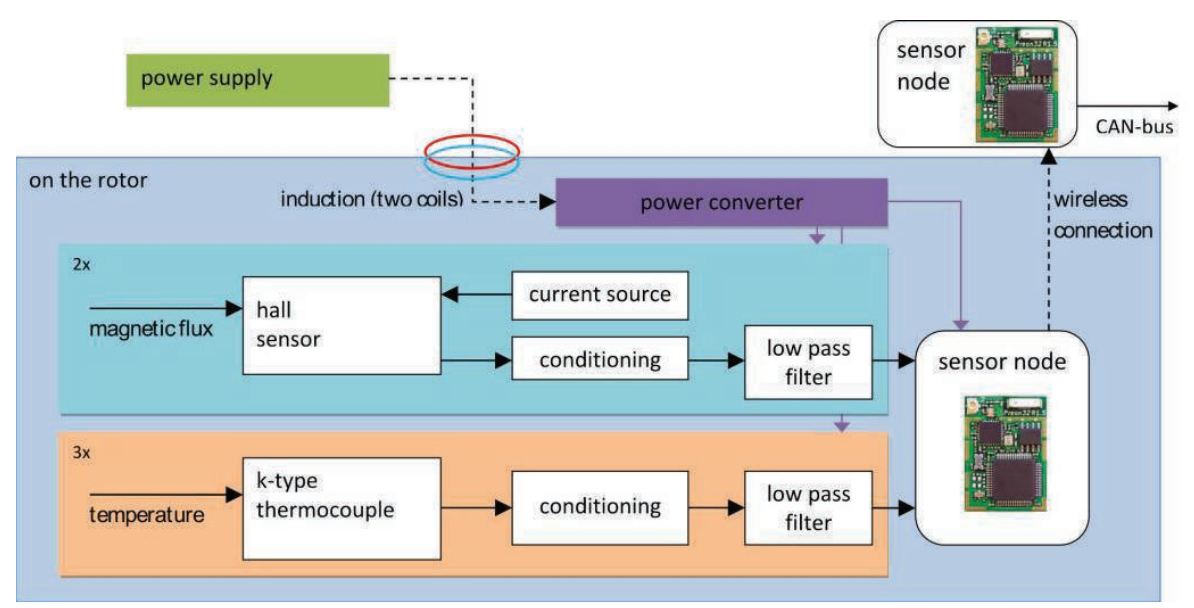

Fig. 2. Structure of the wireless sensor

\subsection{Inductive Power Supply}

The inductive power supply used for the wireless sensor follows the general design described in [3]. Two coils are placed coaxially to the motor's axis of rotation. The primary coil is fixed on the motor casing. The secondary coil is fixed on the rotor. Together the two coils form a transformer circuit. Thus, a sinusoidal supply voltage can be transmitted from the outside to the sensor, regardless of whether the motor is rotating or not.

To maximize the magnetic coupling, and thus the efficiency of the transmission, the two coils where placed in a way that the primary coil is partly inside the secondary coil (see Fig. 4). To minimize its weight and to avoid a reverse magnetic flux within the area enclosed by the secondary windings, the secondary coil holder is made out of plastic using a 3D-printer. To keep the generator circuit simple and to reduce eddy current losses as well as the risk of unwanted electromagnetic radiation, the coils were designed for a sinusoidal input voltage with a frequency in the range of $1-10 \mathrm{kHz}$.

The implemented generator circuit can be connected to a standard $12 \mathrm{~V} \mathrm{DC}$ power supply. A control loop ensures a constant RMS-current through the primary coil. This enables the generator to compensate changes in the coils resistance due to temperature variations. On the secondary side a supply voltage of 3.3 V DC for the sensor is generated by a full-bridge rectifier and a linear voltage regulator. On both, the primary and the secondary side capacitors are placed in series to the coils in order to minimize reactive currents.

Experiments were done to characterize the inductive power transmission and to optimize the frequency used (see section 5.1).

\subsection{Wireless Sensor}

The basis for the wireless sensor is a Preon32 radio module from Virtenio $\mathrm{GmbH}$. It contains a 32 bit ARM microcontroller with a $2.4 \mathrm{GHz}$ wireless transceiver compliant to the IEEE 802.15.4 standard. The microcontroller provides two 12 bit analog-to-digital converters (ADC) with a maximum sampling rate of $1 \mathrm{MSamples} / \mathrm{s}$ that can be used to digitize analog sensor data.

\subsection{Analog Data Acquisition}

Three k-type, class 2 thermocouples were used to measure the temperature on the rotor. Their signals are amplified by a single-chip thermocouple amplifier with integrated cold junction compensation and fed into the anti-aliasing filters described below. Given the tolerances of the thermocouples and the sensor circuitry the overall accuracy of the temperature measurement was calculated to be $3.5 \mathrm{~K}$. A semiconductor temperature sensor is used to monitor the temperature on the printed circuit board.

To measure the magnetic flux density two analog hall sensors, type HE144 from Hoeben Electronics, are used. They are driven by a $100 \mu \mathrm{A}$ constant current source and the resulting hall voltage is amplified by an instrumentation amplifier.

Fig. 3 gives an overview of the measurement chain used in the wireless sensor. For each of the 5 analog sensor channels ( 3 thermocouples +2 hall sensors) a $4^{\text {th }}$ order analog anti-aliasing filter was implemented. The filters have a butterworth characteristic and a cutoff frequency of $10 \mathrm{~Hz}$.

All sensor channels are sampled with a sampling rate of 200 Samples/s each. On the Preon32 the sampling rate is digitally decimated to $25 \mathrm{Samples} / \mathrm{s}$. This enables a more efficient use of the available bandwidth in the wireless transmission channel. 


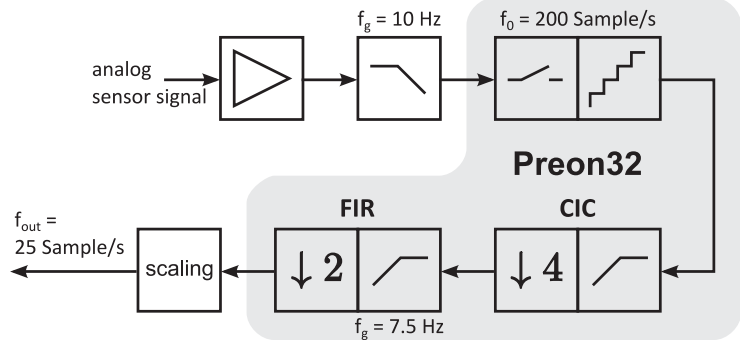

Fig. 3. Measurement chain used in the wireless sensor

For the decimation a cascade of cascadedintegrator-comb $(\mathrm{ClC})$ and finite impulse response (FIR) filters is used. This way a sharp cutoff in the overall magnitude frequency response can be achieved without the need for higher order analog filters. CIC filters have the advantage of providing high decimation rates at only little computational cost [4]. Their major drawback is a non-constant magnitude frequency response in the pass band. To compensate for this FIR filters with complementary frequency responses are used in the last stage (see [5]). The overall $3 \mathrm{~dB}$ bandwidth of the measurement chain is $7.5 \mathrm{~Hz}$.

The scaling coefficients of the sensors are stored directly on the sensor node, making it possible to convert the measured values to SIunits before transmission.

\subsection{Sensor Software}

Both Preon32 sensor nodes, the one on the wireless sensor as well as the one connected to the CAN-bus of the test bench where programmed in $\mathrm{C}$ using the Contiki operating system [6]. Contiki is specifically optimized towards the needs of wireless sensor networks and includes amongst other things a complete IPv6 based communication stack. It is designed for the use in wireless sensor networks which makes it easy to extend the system with additional wireless sensors in the future.

The operation of the sensor software can be summarized as follows: as soon as the node on the rotor is powered on it starts acquiring data. The ADC samples a block of 8 samples per channel and writes it into one of two buffers using direct memory access (DMA). Every time one of the buffers is full, the ADC immediately goes on to write to the other buffer, while the processor filters the samples. This way a truly continuous data acquisition can be achieved. After one block of samples has been filtered the results are immediately transmitted to the node connected to the CAN-bus. Every wireless packet contains exactly one sample per channel as well as the timestamp at which they were sampled by the ADC.
Once the CAN-node receives a wireless packet it immediately relays the data to the CAN-bus. For each channel one CAN-message is generated containing the measurement value in millitesla or degrees centigrade as a 32 bit floating point number as well as the corresponding timestamp in seconds.

\subsection{Mechanical Integration}

To gain enough space for the sensor between the backside casing of the motor and the rotor a spacer ring was installed between the front- and backside of the motor casing. In order to keep the same distance between the position encoder and the shaft-end magnet a new bearing holder was manufactured that at the same time holds the primary coil of the inductive power supply. Fig. 4 shows the rotor and modified backside of the motor casing.

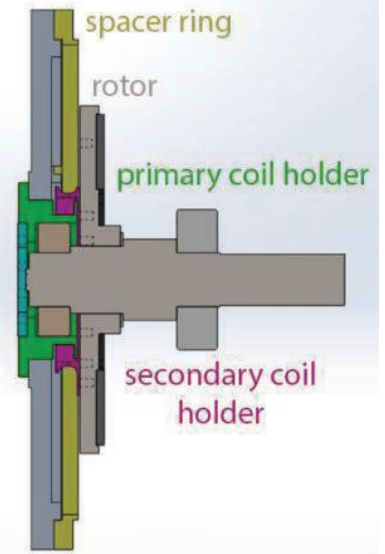

Fig. 4. Modified backside parts of the axial flux machine

For the thermocouples three small holes were drilled into the rotor back iron. All holes are located on one radial line at different distances from the center. The thermocouples were fixated in the holes using heat paste and epoxy adhesive. One hall sensor was glued to a magnet directly below the thermocouples. The second was glued to another magnet. Fig. 5 shows a cross-section of the rotor with the installed sensors.

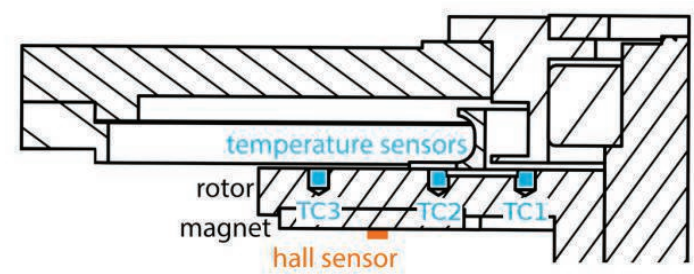

Fig. 5. Locations of sensors on the rotor

The printed circuit board (PCB) containing the sensor circuits as well as the secondary coil holder were fixated on the backside of the rotor using screws. Fig. 6 shows the sensor PCB and secondary coil holder installed on the rotor. 


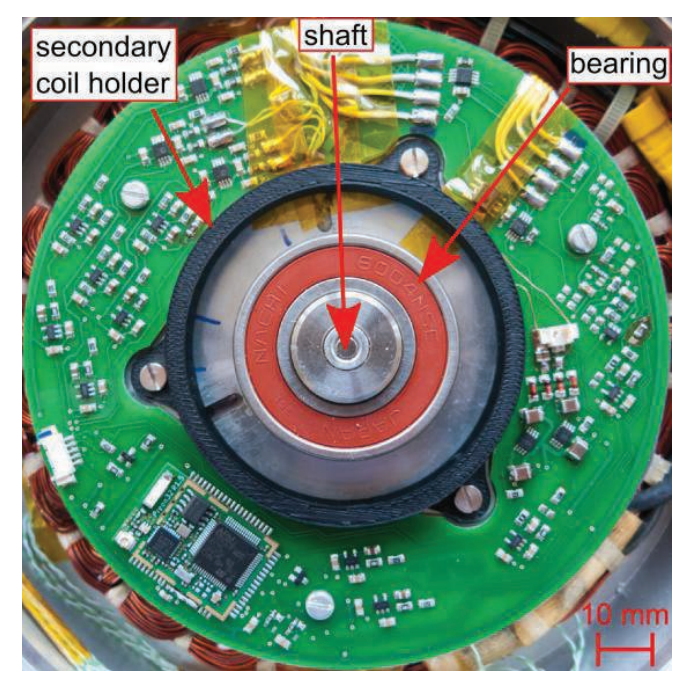

Fig. 6. PCB with sensor circuit and secondary coil mounted on the rotor

\section{Experiments}

\subsection{Characterization of Power Supply}

To find the optimum operating conditions for the power supply the input frequency was varied in the range of $1-16 \mathrm{kHz}$. At each frequency the primary voltage and current, that were needed to sustain a load current of $30 \mathrm{~mA}$ at $3.3 \mathrm{~V}$ required to guarantee the operation of the sensor on the secondary side, were measured. It was found that the efficiency of the transmission is increasing with the frequency. At frequencies higher than $3 \mathrm{kHz}$ this increase is only marginal, while the required primary voltage is rising more significantly, making the design of the generator circuit more difficult. Thus, it was decided to operate at a frequency of $3 \mathrm{kHz}$. At this point a primary RMS current of $256 \mathrm{~mA}$ with a primary RMS voltage of $5 \mathrm{~V}$ can supply a constant secondary load with $34 \mathrm{~mA}$ at $3.3 \mathrm{~V}$. The overall efficiency of the transmission is about $8 \%$.

\subsection{Calibration of Flux Sensors}

The flux sensor were calibrated at 6 points in the range of -1 to $1 \mathrm{~T}$ using an electromagnet and a gauss meter (F.W. Bell 9900, accuracy $0.035 \% \pm 11$ Counts) as a reference. Taking into account the tolerances of the calibration as well as the sensor circuitry the overall accuracy of the magnetic flux density measurement was calculated to be $17 \mathrm{mT}$.

\subsection{Test in climatic chamber}

The sensor installed in the motor was tested in a climatic chamber over a temperature range of 20 to $100^{\circ} \mathrm{C}$. The sensor circuit was found to operate without problems. All temperature sensor readings were equal to each other within their calculated accuracy and matched well with the temperature displayed by the climatic chamber. The measured change in magnetic flux density matched well with the specified temperature coefficient of the magnetic material.

\subsection{Measurements at Motor Test Bench}

Finally the motor with the wireless sensor was installed on the test bench introduced in section 3.2. The CAN-node was placed at a distance of about $0.4 \mathrm{~m}$ from the motor. No problems were observed with the wireless communication. First experiments were run to verify that the sensor is operating correctly.

Fig. 7 shows the results from two preliminary experiments with fixed motor speed. In both cases, the motor output torque is shown in the top graph, the measured magnetic flux is shown in the second graph whilst stator coil and rotor temperatures are compared in the third graph. In fig. $7 a$, the slow transient behavior of the motor at $4500 \mathrm{rpm}$ is presented, giving an indication of the temperatures that would be observed during continuous operation. In fig. $7 \mathrm{~b}$, a rapid load transient at $500 \mathrm{rpm}$ highlights the differences in thermal inertia that are important for transient duty cycles. The aim here is to contrast two styles of motor usage: continuous low torque operation to assist cruising and torque addition for transients.

For the $4500 \mathrm{rpm}$ transient (Fig. 7a), the maximum torque that is achieved during the $45 \mathrm{~min}$ loading period is $3.5 \mathrm{Nm}$ which is the continuous torque of the motor. Under these conditions the motor is designed to operate continuously. For the $500 \mathrm{rpm}$ transient (Fig. 7b), the maximum torque is $9.5 \mathrm{Nm}$ which is considerably higher than the continuous torque. Because of the significant heat generated by the high current flowing in the coils, the motor can only operate in these conditions for a limited period (in this case around $45 \mathrm{~s}$ ). After the torque step the motor is allowed to cool down whilst being spun by the test cell dynamometer. During this period a small negative torque is recorded corresponding to the motor friction.

The effect of temperature on the magnetic flux is apparent, especially in fig $7 a$. As the temperature of the rotor increases, the magnetic flux decreases, in this case by around $55 \mathrm{mT}$ for a measured $75^{\circ} \mathrm{C}$ increase in rotor temperature. The effect is seen to be reversible during this experiment as the magnetic flux increases again during the cooling down period, however if excessive magnet temperatures incurred, a permanent demagnetization could be caused. Other variations in magnetic flux can be observed, notably in fig. $7 \mathrm{~b}$ which are a result of changes in motor currents but these are beyond the scope of this paper. 

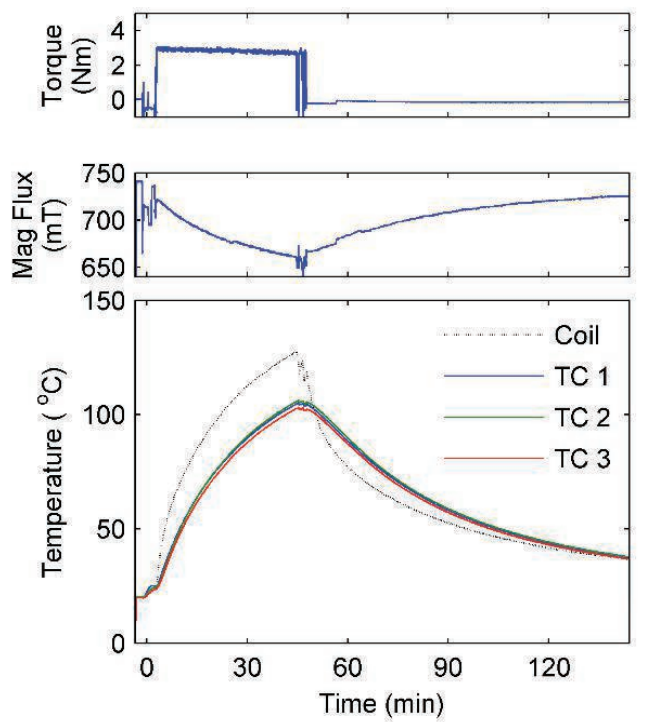

(a) 4500 rpm Slow Continuous Load Transient
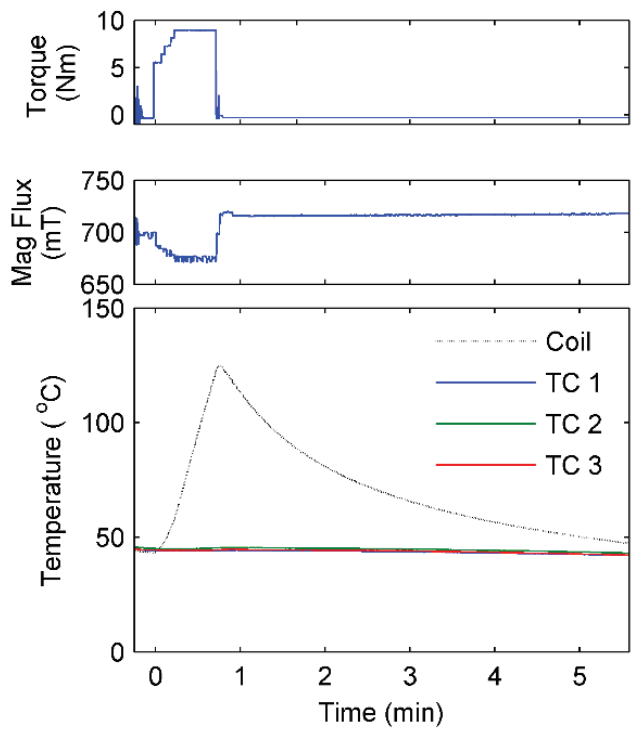

(b) 500 rpm Rapid Rated Load Transient

Fig. 7. Torque, temperatures and magnetic flux density measured on the rotor and stator coil

Considering now the temperature measurements from each experiment: in both cases it is obvious that the temperature of the coils rises faster than that of the rotor. This is easily explained by the fact that the coils are both a major heat source and have a relatively small thermal inertia. This is most extreme in the rapid $500 \mathrm{rpm}$ transient (Fig. 7b) where the coil temperature increases rapidly from $45^{\circ} \mathrm{C}$ to $125^{\circ} \mathrm{C}$ in around $45 \mathrm{~s}$ without any significant change in the measured rotor temperatures. In contrast, the rated torque/speed condition in fig. 7a shows a significant build-up of heat within the rotor, with a temperature rise from $25^{\circ} \mathrm{C}$ to $100{ }^{\circ} \mathrm{C}$ of the rotor whilst the coil temperature increases $25^{\circ} \mathrm{C}$ to $125^{\circ} \mathrm{C}$. During the cooling down period (45 min-130 $\mathrm{min}$ ), the rotor temperature is hotter than the coil temperature owing to thermal inertia and internal heat flows. Further experiments at various conditions will permit the validation of thermal models to improve hybrid control strategies.

\section{Conclusions and Outlook}

A wireless sensor that measures the temperatures and magnetic flux density at the rotor of an axial flux permanent magnet machine was successfully designed and implemented. An inductive power supply makes it possible to run the system continuously without ever having to change batteries. The hall sensors of the sensor were calibrated using a reference probe and the entire sensor was successfully tested in a climatic chamber up to a temperature of $100{ }^{\circ} \mathrm{C}$. The motor with the sensor was installed at a test bench. The data of the wireless sensor was relayed to the test bench's CAN-bus. First experiments show plausible results.
The wireless sensor enables the measurement of quantities in the motor that were previously inaccessible. This new information will be used to build better thermal models of the motor. Those will enable the development more effective thermal management strategies that have the potential of significantly improving the performance of electric and hybrid electric vehicles. Due to the design of the sensor it is easy to add additional wireless sensors in the future if required.

\section{References}

[1] S. C. Oh, A. Emadi, Test and simulation of axial flux motor characteristics for hybrid electrical vehicles, IEEE Transactions on Vehicular Technology, 53, 912-919 (2004), doi: 10.1109/TVT.2004.827165

[2] J. Funck, R. Knoblich, D. Scholz, S. Nowoisky, C. Gühmann, Drahtloses Messsystem für Temperaturen in Kraftfahrzeugkupplungen, Technisches Messen, 80, 84-91 (2013), doi: 10.1524/teme.2013.0014

[3] P. Burisch, Induktive Energieversorgung eines drahtlosen Temperaturmesssystems, Diplomarbeit, TU Berlin, 2014

[4] E. Hogenauer, An economical class of digital filters for decimation and interpolation, IEEE Transactions on Acoustics, Speech and Signal Processing, 29, 155-162 (1981), doi: 10.1109/TASSP.1981.1163535

[5] Altera Corp., Understanding CIC Compensation Filters, Application Note 455, (2007), url: http://www.altera.com/literature/an/an455.pdf (accessed: 2015-02-06)

[6] Contiki: The Open Source OS for the Internet of Things, http://www.contiki-os.org/ (accessed: 2015-02-06) 